\title{
Novel three-body nano-abrasive wear mechanism
}

\author{
Ruling CHEN*, Shaoxian LI \\ College of Mechanical Engineering, Donghua University, Shanghai 201620, China \\ Received: 01 July 2020 / Revised: 07 October 2020 / Accepted: 07 December 2020 \\ (C) The author(s) 2020.
}

\begin{abstract}
Current three-body abrasive wear theories are based on a macroscale abrasive indentation process, and these theories claim that material wear cannot be achieved without damaging the hard mating surface. In this study, the process of three-body nano-abrasive wear of a system including a single crystalline silicon substrate, an amorphous silica cluster, and a polyurethane pad, based on a chemical mechanical polishing (CMP) process, is investigated via molecular dynamics simulations. The cluster slid in a suspended state in smooth regions and underwent rolling impact in the asperity regions of the silicon surface, realizing non-damaging monoatomic material removal. This proves that indentation-plowing is not necessary when performing CMP material removal. Therefore, a non-indentation rolling-sliding adhesion theory for three-body nano-abrasive wear between ultrasoft/hard mating surfaces is proposed. This wear theory not only unifies current mainstream CMP material removal theories, but also clarifies that monoatomic material wear without damage can be realized when the indentation depth is less than zero, thereby perfecting the relationship between material wear and surface damage. These results provide new understanding regarding the CMP microscopic material removal mechanism as well as new research avenues for three-body abrasive wear theory at the monoatomic scale.
\end{abstract}

Keywords: wear mechanism; material removal mechanism; three-body abrasive wear; chemical mechanical polishing (CMP); molecular dynamics simulation

\section{Introduction}

Over the past three decades, the three-body abrasive wear mechanism has not developed significantly, although many reports regarding the applications of three-body abrasive wear have been published annually. Three-body abrasive wear [1-3], in which abrasive move between the two mating surfaces of a friction couple, has been investigated for approximately 60 years [4] because wear and friction constitute approximately $20 \%$ of energy consumption in daily life $[5,6]$. The current three-body abrasive wear mechanism presented in textbooks primarily includes the indentation rolling-sliding plastic deformation theory $[7,8]$ and indentation-cutting theory $[1,4,9]$. For the former, the two mating surfaces of a friction couple are both hard, whereas the indentation depths of abrasives penetrating the mating surface are relatively small. Hence, the abrasives tend to roll on the mating surface, inducing plastic deformation on the mating surface $[10,11]$. For the latter, one matching surface is soft, whereas the other is hard. The abrasives become trapped in the soft surface and penetrate the hard surface with relatively large indentation depths. Hence, the abrasives are more susceptible to sliding along the hard mating surface $[12,13]$. The damage mechanism of the hard mating surface is primarily based on the indentation-cutting process, accompanied by plastic deformation [13, 14]. Current three-body abrasive theories are based on the abrasive indentation process at the macroscale. According to these theories, when the indentation depth is equal to zero, no material is removed on the hard mating surface $[15,16]$. In other words, material wear cannot be achieved without

* Corresponding author: Ruling CHEN, E-mail: chen_ruling@dhu.edu.cn 
damaging the hard mating surface.

Three-body nano-abrasive wear between ultrasoft/ hard mating surfaces is a new process for three-body abrasive wear, but no wear theory suitable for this configuration has been proposed hitherto. Three-body nano-abrasive wear between ultrasoft/hard mating surfaces is defined as the movement of a nano-abrasive between ultrasoft and hard mating surfaces. Owing to the rapid development of nanotechnology in the past three decades, three-body nano-abrasive wear between ultrasoft/hard mating surfaces has become increasingly important, not only for energy saving, but also for ultraprecision machining. The existing indentation-cutting theory between soft/hard mating surfaces is typically applied directly to this new situation, resulting in a gap between practical applications and theoretical research.

Chemical mechanical polishing (CMP) technology is a typical application of the new wear process described above. Moreover, the microscopic material removal mechanism of CMP is yet to be elucidated. CMP is the most widely accepted method to achieve global planarization in the fabrication of integrated circuits (ICs) [17]. During CMP, a small amount of slurry containing nano-abrasives (e.g., amorphous silica abrasives with diameters of 10-100 nm [18]) is added to the interface between an ultrasoft polyurethane pad with an elastic modulus of 100-500 MPa $[18,19]$ and a hard wafer (e.g., a crystalline $\mathrm{Si}$ (001) wafer with an elastic modulus of approximately $130 \mathrm{GPa}$ [20]) to achieve ultrasmooth wafer machining surfaces [21]. The line widths of ICs have now been reduced to less than $7 \mathrm{~nm}$, and the understanding of the material removal mechanism of CMP has become extremely urgent. The current mainstream model of CMP material removal theory is the indentation-plowing theory [21-25] based on the indentation-cutting theory of three-body abrasive wear. In this model, nano-abrasives are trapped and fixed in an ultrasoft polishing pad; subsequently, they penetrate the hard wafer surface. The abrasives then slide along the wafer surface to realize material removal on the wafer surface by the plowing effect. However, the validity of indentationplowing theory has been questioned in the past three decades because it cannot explain experimental observations where almost no scratches appear during a normal CMP. Moreover, owing to the different interpretations of the contact status between the abrasives and pad, indentation-plowing theory is controversial [26], and the models proposed by Luo and Dornfeld [23] and Zhao and Chang [25] have been presented. In this paper, these two models are abbreviated as Luo model [23] and Zhao model [25], respectively. Therefore, scholars have proposed the abrasive-impact theory [27-29]. In the abrasive-impact model, nanoabrasives collide with the wafer surface during CMP to achieve material removal. However, abrasive-impact theory cannot explain the mechanism by which the abrasives obtain relatively high initial impact energy.

We believe that the aforementioned discrepancies in the understanding of the CMP material removal mechanism are caused by the failure to fully understand the role of the pad during material removal. In indentation-plowing theory, the ultrasoft pad is simplified as a normal soft pad, whereas in abrasiveimpact theory, the ultrasoft pad is disregarded. Therefore, a molecular dynamics simulation (MD/MDS) model that included a polyurethane pad, an amorphous silica cluster, and a hard crystal silicon plate was established in this study. To the best of our knowledge, this model mimics the actual CMP the closest, and it is the first model of three-body abrasive wear between ultrasoft/hard mating surfaces.

\section{Molecular dynamics simulation methodology}

\subsection{Simulation model}

As shown in Fig. 1, the polyurethane pad in this study was simulated by adopting a beard-spring chain model, and each chain contained six monomers [30]. The relative atomic mass of the monomer was 59 based on the generic structure of polyurethane [31]. This polyurethane pad comprised 55,296 monomers, and it measured $234.85 \AA(x) \times 234.85 \AA(y) \times 69.21 \AA$ $(z)$. The nominal elastic modulus and hardness of this pad were approximately 1.016 and $0.107 \mathrm{GPa}$, respectively.

The upper and lower single crystal silicon plates in the simulation model represent the wafer and platen in the real CMP, respectively. They were initially arranged in a diamond cubic structure with a lattice constant of $5.43 \AA$ and a crystal orientation of (001). 


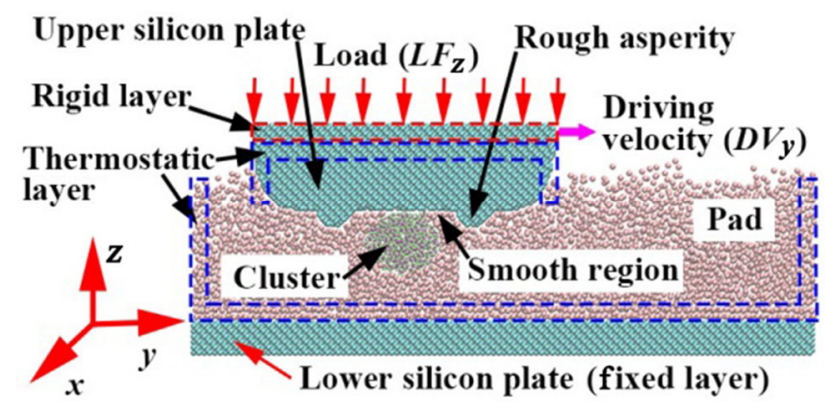

Fig. 1 Simulation models of ultrasoft/hard three-body nanoabrasive wear among single crystal silicon plate, polyurethane pad, and amorphous silica cluster.

Their dimensions were $152.04 \AA(x) \times 152.04 \AA(y) \times$ $43.44 \AA(z)$ and $234.85 \AA(x) \times 234.85 \AA(y) \times 16.29 \AA$ $(z)$, respectively. Moreover, two asperities were observed on the surface of the upper silicon plate, both of which were composed of semi-cylinders of $18 \AA$ in diameter. Finally, an amorphous silica cluster, which comprised 1,536 atoms with a diameter of approximately $36 \AA$, represented an abrasive particle during CMP.

The interatomic interactions in the silica clustersilicon plate system were modeled by a StillingerWeber-like potential [32]. For the two adjacent bonding pad monomers in the same chain, the interactions between the monomers were described by the finitely extended nonlinear elastic (FENE) potential [26, 30]. The interactions among the nonbonding pad monomers, pad-silicon plates, and pad-silica clusters were described by the 12-6 Lennard-Jones potential $[26,30]$.

In the simulation, the lower silicon substrate was fixed. The top layer of the upper silicon plate with $10 \AA$ in thickness was treated as a rigid body. Both the working load $\left(L F_{z}\right)$ and the horizontal driving speed $\left(D V_{y}\right)$ applied during the simulation acted on this rigid layer. The upper area near the rigid layer of the upper silicon plate, the peripheries of the upper silicon plate and the pad, and the bottom area of the pad were set to thermostatic layers of $10 \AA$ in thickness. The temperature of the thermostatic layer was maintained at $293 \mathrm{~K}$ using the Gauss-constraint method [33]. Before the upper silicon plate started to move horizontally, an initial vertical loading process occurred for 200,000 fs, which was sufficient to achieve an initial equilibrium state among the silicon plate, cluster, and pad. In this study, the simulation timestep was set to $1 \mathrm{fs}$. Details regarding the simulation of the initial vertical loading process are available in our previously Ref. [26] or Fig. S1 in the Electronic Supplementary Material (ESM).

\subsection{Identification of contact status}

When the cluster was located below the smooth region of the upper silicon plate, the upper silicon plate atoms measuring $30 \AA \times 30 \AA \times 43.44 \AA$ above the cluster were analyzed, as shown in Fig. 2 . The average value of the first 10 minimum $z$-coordinate values of these selected silicon atoms was used as the height of the smooth region of the upper silicon plate $\left(h_{1}\right)$. Meanwhile, the average value of the top 10 maximum $z$-coordinate values in the cluster atoms was used as the height of the cluster vertices $\left(h_{2}\right)$. The difference between $h_{1}$ and $h_{2}$ was defined as the spacing $(\Delta h)$ between the cluster and the smooth region of the silicon plate, as shown in Fig. 2(a).

When the cluster was located below the asperity of the upper silicon plate, the distances $(l)$ between the cluster atoms that did not disperse and the center of the cylindrical asperity was calculated first. The normal distance from each cluster atom to the asperity surface was obtained by subtracting the radius $(r)$ of the semi-cylindrical asperity. Finally, the average value of the first 10 minimum normal distances was used as
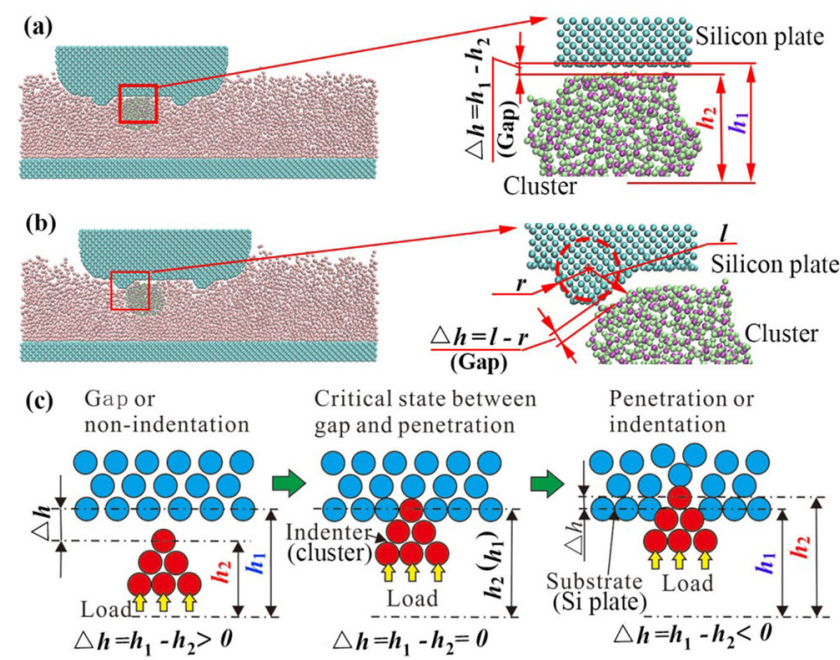

Fig. 2 Schematic illustration of contact status between cluster and upper silicon plate. (a) Cluster and smooth region of upper silicon plate are separated by a gap. (b) Cluster and asperity region of upper silicon plate are separated by a gap. (c) As spacing changed from positive to negative, contact status between cluster (indenter) and silicon plate (substrate) changed from a gap or non-indentation state to a penetration or indentation state. 
the spacing $(\Delta h)$ between the cluster and the asperity surfaces of the silicon plates, as shown in Fig. 2(b).

When the spacing $(\Delta h)$ was greater than zero, a gap appeared between the cluster and the surface of the silicon substrate, as shown in Fig. 2(c). Conversely, when the spacing was less than zero, the cluster penetrated the surface of the silicon substrate. In other words, as the spacing decreased gradually from a positive value to a negative value, the contact status between the cluster and silicon plate changed correspondingly from a non-indentation state to an indentation state.

\section{Simulation results}

\subsection{Contact status between cluster and hard substrate}

First, the most significant difference between our current findings and the existing three-body abrasive wear theories is that a gap is always present between the cluster and the upper silicon plate, whether in the smooth or the rough driving stage (Fig. 3). Hence, the cluster did not penetrate the silicon plate but was suspended on the surface of the silicon plate in a non-indentation form. However, according to the existing three-body abrasive wear theory $[1,4]$ or the CMP indentation-plowing model [23, 25], the abrasive should have penetrated the wafer surface during CMP, as shown in Fig. 3(c).

The gap was generated by the relatively low local pressure and local temperature of the upper silicon plate in the contact zone. Compared with the pad, the proportion of the operating load imposed on the cluster was small, i.e., less than $5 \%$ during the driving process, as shown in Fig. S2 in the ESM. Hence, the local pressure of the silicon plate in the contact zone between the cluster and upper silicon plate was much smaller than the surface hardness of the upper silicon plate. As shown in Figs. S3 and S4 in the ESM, under an operating load of $75 \mathrm{nN}$, the maximum local pressure and local temperature in the contact area were approximately $8.3 \mathrm{GPa}$ and $500 \mathrm{~K}$, respectively. This maximum local pressure was significantly less than the normal hardness of the $c$-Si(001) substrate, i.e., approximately 11.35 GPa [34]. Therefore, the cluster
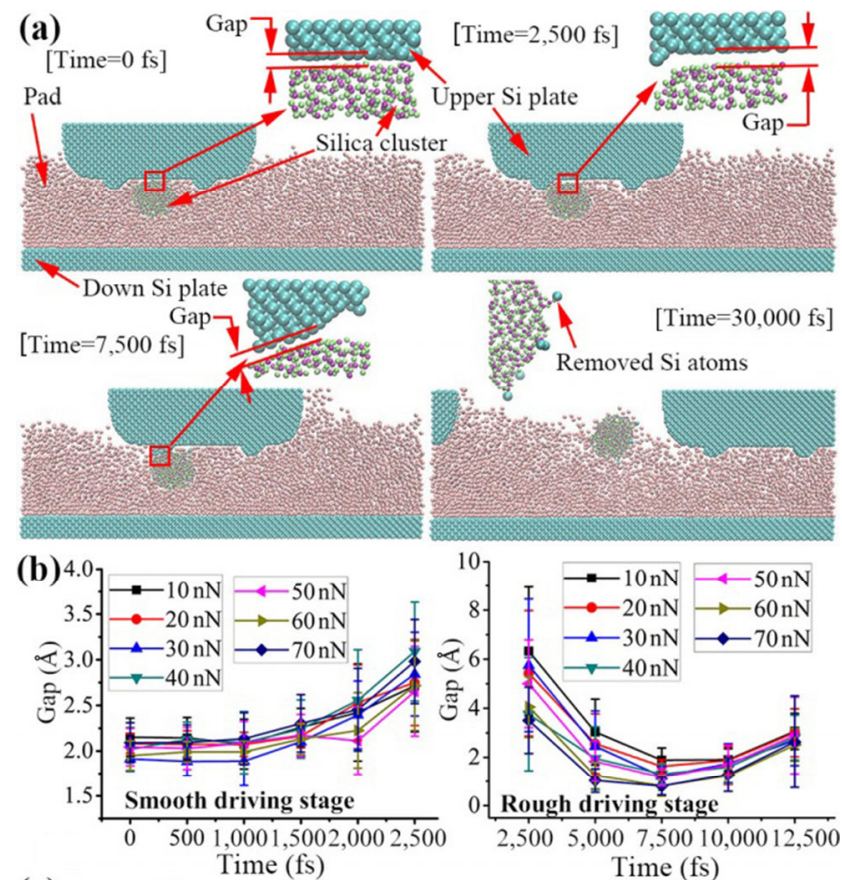

(c)

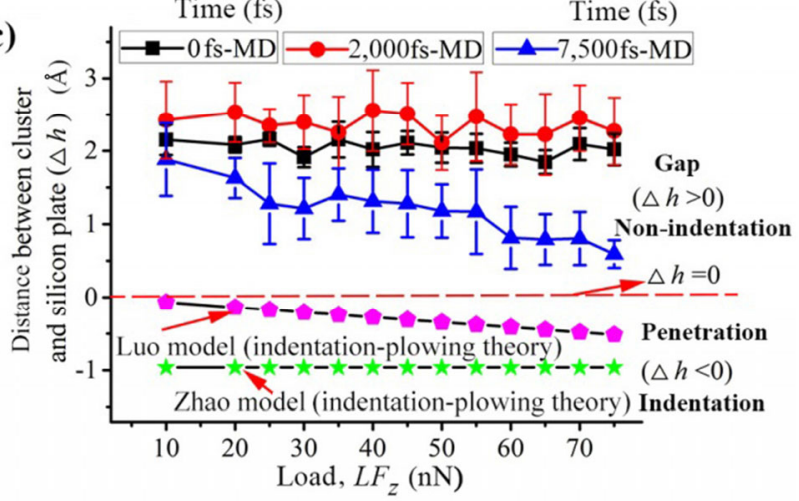

Fig. 3 Analysis of gap between cluster and upper silicon plate during driving at driving velocity of $500 \mathrm{~ms}^{-1}$. (a) Snapshots of atomic positions and corresponding enlarged views of gap at different moments under working load of $40 \mathrm{nN}$. The system was in smooth driving stage at 0 and 2,000 fs. At 7,500 and 30,000 fs, the system was in rough driving and separation stages, respectively. (b) Simulation results of gap during smooth and rough driving stages under different working loads. (c) Comparisons of gap results from existing indentation-plowing theory and by MD of this study.

could not penetrate the surface of the upper silicon plate during driving.

\subsection{Movement status of cluster}

The second interesting finding is that although the cluster is completely embedded in the soft polishing pad, it can still undergo rolling and sliding (Fig. 4), unlike the claims of the existing indentation-cutting 

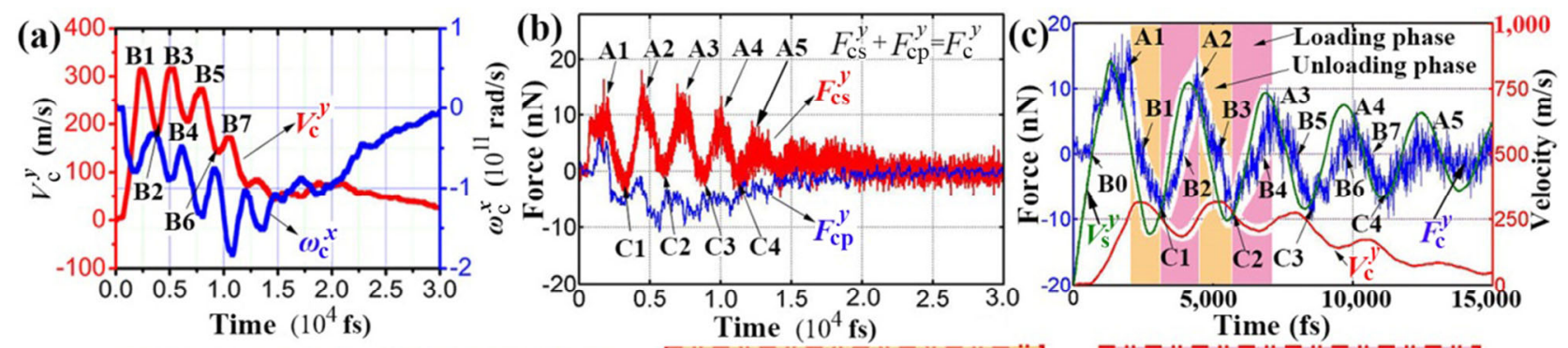

(d)
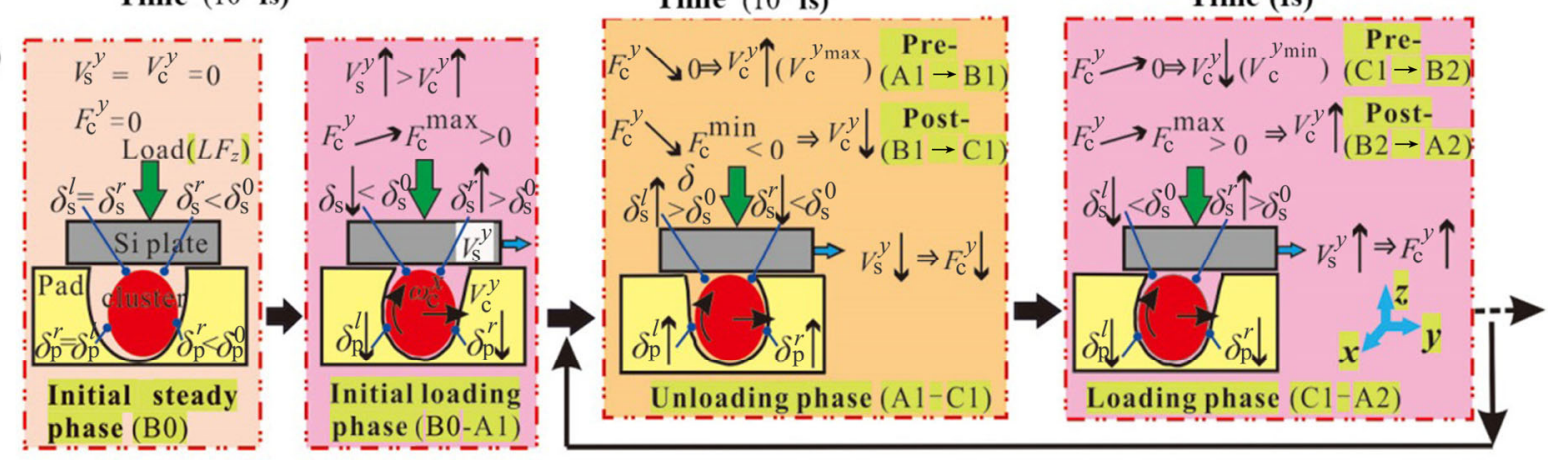

Fig. 4 Analysis of velocity, force, and motion of cluster during driving under $L F_{z}=40 \mathrm{nN}$ and $D V_{y}=500 \mathrm{~ms}^{-1}$. (a) $y$-direction centroid translational velocity $\left(V_{\mathrm{c}}^{y}\right)$ and $x$-direction centroid rotational velocity $\left(\omega_{\mathrm{c}}^{x}\right)$ of cluster. (b) Action force on silica cluster in $y$-direction $\left(F_{\mathrm{c}}^{y}\right) . F_{\mathrm{cs}}^{y}$ and $F_{\mathrm{cp}}^{y}$ represent forces acting on cluster by upper silicon plate and pad, respectively. (c) Analyses of periodicities of velocities and forces of cluster. In the loading and unloading phases, $F_{\mathrm{c}}^{y}$ increased and decreased gradually, respectively. $F_{\mathrm{c}}^{y}$ underwent maxima at $\mathrm{A} 1, \mathrm{~A} 2, \mathrm{~A} 3, \mathrm{~A} 4$, and $\mathrm{A} 5$, and minima at $\mathrm{C} 1, \mathrm{C} 2, \mathrm{C} 3$, and $\mathrm{C} 4$, respectively. $F_{\mathrm{c}}^{y}$ was zero at $\mathrm{B} 0, \mathrm{~B} 1, \mathrm{~B} 2, \mathrm{~B} 3, \mathrm{~B} 4, \mathrm{~B} 5, \mathrm{~B}$, and B7. (d) Schematic diagram of rolling-sliding motion with stick-slip characteristics of cluster trapped in pad during driving. $\delta_{\mathrm{s}}^{0}$ and $\delta_{\mathrm{p}}^{0}$ denote gaps when forces between cluster and upper silicon plate $\left(F_{\mathrm{cs}}\right)$ and between cluster and pad $\left(F_{\mathrm{cp}}\right)$ were zero, respectively.

theory. Both the centroid translational velocity $\left(V_{c}^{y}\right)$ and centroid rotational angular velocity $\left(\omega_{\mathrm{c}}^{x}\right)$ of the cluster increased significantly during the smooth and rough region driving stages, as shown in Fig. 4(a). The translational and rotational velocities were synchronized. However, the current indentation-cutting theory of three-body abrasive wear between soft/hard mating surfaces or CMP indentation-plowing theories suggest that the abrasives will be trapped in the pad or soft substrate and should be stationary.

Furthermore, the rolling-sliding behavior of the cluster trapped in the pad showed stick-slip characteristics, unlike the claims of the current indentation rolling-sliding plastic deformation theory. First, the movement of the cluster trapped in the pad was similar to the forward peristalsis of earthworms through muscle contraction and relaxation, as shown in Fig. 4(d). As the upper silicon plate was pushed horizontally from the left to the right, after the initial steady and initial loading phases, the movement status of the cluster entered a cyclic change between the unloading and loading phases. Accordingly, the left and right gaps between the cluster and pad ( $\delta_{\mathrm{p}}^{l}$ and $\delta_{\mathrm{p}}^{r}$, respectively), the left and right gaps between the cluster and upper silicon plate $\left(\delta_{\mathrm{s}}^{l}\right.$ and $\delta_{\mathrm{s}}^{r}$, respectively), the cluster's horizontal resultant force $\left(F_{c}^{y}\right)$, and the cluster's centroid translational velocity $\left(V_{c}^{y}\right)$ changed periodically. Moreover, the period between $V_{c}^{y}$ and $F_{c}^{y}$ was the same, but a $90^{\circ}$ phase difference was observed between them. Furthermore, the average movement distance of the cluster centroid during each cycle in the $y$-direction $\left(S_{0}\right)$ was approximately $5.478 \AA$, which was similar to the lattice constant of a single-crystal silicon (5.43 $\AA$ ). Therefore, the rolling-sliding process with stick-slip characteristics differed significantly from the continuous rolling-sliding characteristics of abrasives in the existing three-body abrasive wear model between hard/hard mating surfaces. More details regarding the analysis of the motion status of the cluster are provided in Figs. S5 and S6 and Table S1 in the ESM.

\subsection{Material removal process}

The third interesting finding is that even though the 
cluster does not penetrate the surface of the upper silicon plate, it can still achieve material removal from the surface of the upper silicon plate. As shown in Fig. 5(a), as the cluster slid at the smooth region and rolled at the asperity region of the upper silicon plate, a few silicon atoms were separated from the surface of silicon plate owing to $\mathrm{Si}-\mathrm{Si}$ bond cleavage. These silicon atoms moved with the cluster by forming a stronger $\mathrm{Si}-\mathrm{O}$ bond between the silicon atom of



(c)

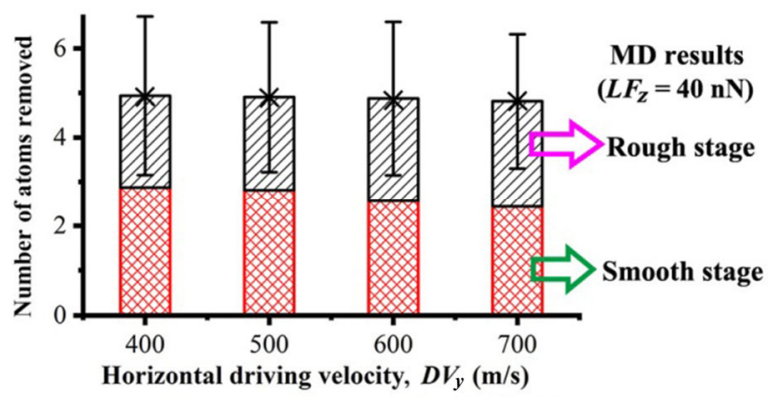

Fig. 5 Material removal process of three-body nano-abrasive wear involving cluster, ultrasoft pad, and hard silicon plate. (a) Silicon atoms of silicon plate were removed, accompanied by formation of atom vacancies, by adhesion between cluster and upper silicon surface under $L F_{z}=40 \mathrm{nN}$ and $D V_{y}=500 \mathrm{~ms}^{-1}$. Monoatomic damage-free material removal on surface of silicon plate was realized. Number and positions of removed atoms in upper silicon plate during driving under different (b) operating loads and (c) driving velocities. silicon plate and the oxygen atoms in the cluster [35, 36]. Meanwhile, a few atomic vacancies formed on the surface of the silicon plate. Therefore, monoatomic material removal on the surface of the silicon plate without surface damage was realized by the adhesion between the cluster and silicon plate. Moreover, the removed silicon atoms were from both the smooth and asperity regions of the upper silicon plate (Fig. 5).

Some experimental reports have provided evidence that material was transferred from the upper silicon plate to the silica cluster. For example, Bun-Athuek et al. [37] experimentally investigated changes in the surfaces and diameters of colloidal silica abrasives during the CMP of sapphire substrates. It was discovered that alumina elements from sapphire substrates dispersed in the used slurry and also aggregated on the surface of the abrasive after polishing when the diameter of the abrasive was $55 \mathrm{~nm}$. Moreover, the alumina elements only aggregated on the surface of the abrasive when the diameter of the abrasive was $20 \mathrm{~nm}$. These results suggested that sapphire can be removed by its adhesion to a silica abrasive during CMP. The simulation results in this study are consistent with the abovementioned experimental phenomena.

\section{Discussion}

\subsection{Non-indentation wear theory}

Based on the findings above, a new non-indentation rolling-sliding adhesion wear theory for three-body nano-abrasive wear between ultrasoft/hard mating surfaces is proposed, as shown in Fig. 6. In this new model, the ultrasoft substrate does not have sufficient grip force to completely fix the abrasive, and the abrasives trapped in the ultrasoft substrate exhibit rolling-sliding behavior with stick-slip characteristics. More importantly, the abrasives cannot penetrate the hard substrate surface but are suspended on the surface of the hard substrate in a non-indentation form. Subsequently, the abrasives slide in a suspended state in the smooth region of the hard substrate and rolling impact will occur at the asperity region of the hard substrate. Finally, the damage-free monoatomic material removal process on the hard substrate surface is achieved by the adhesion between the abrasive and the hard substrate surface. 


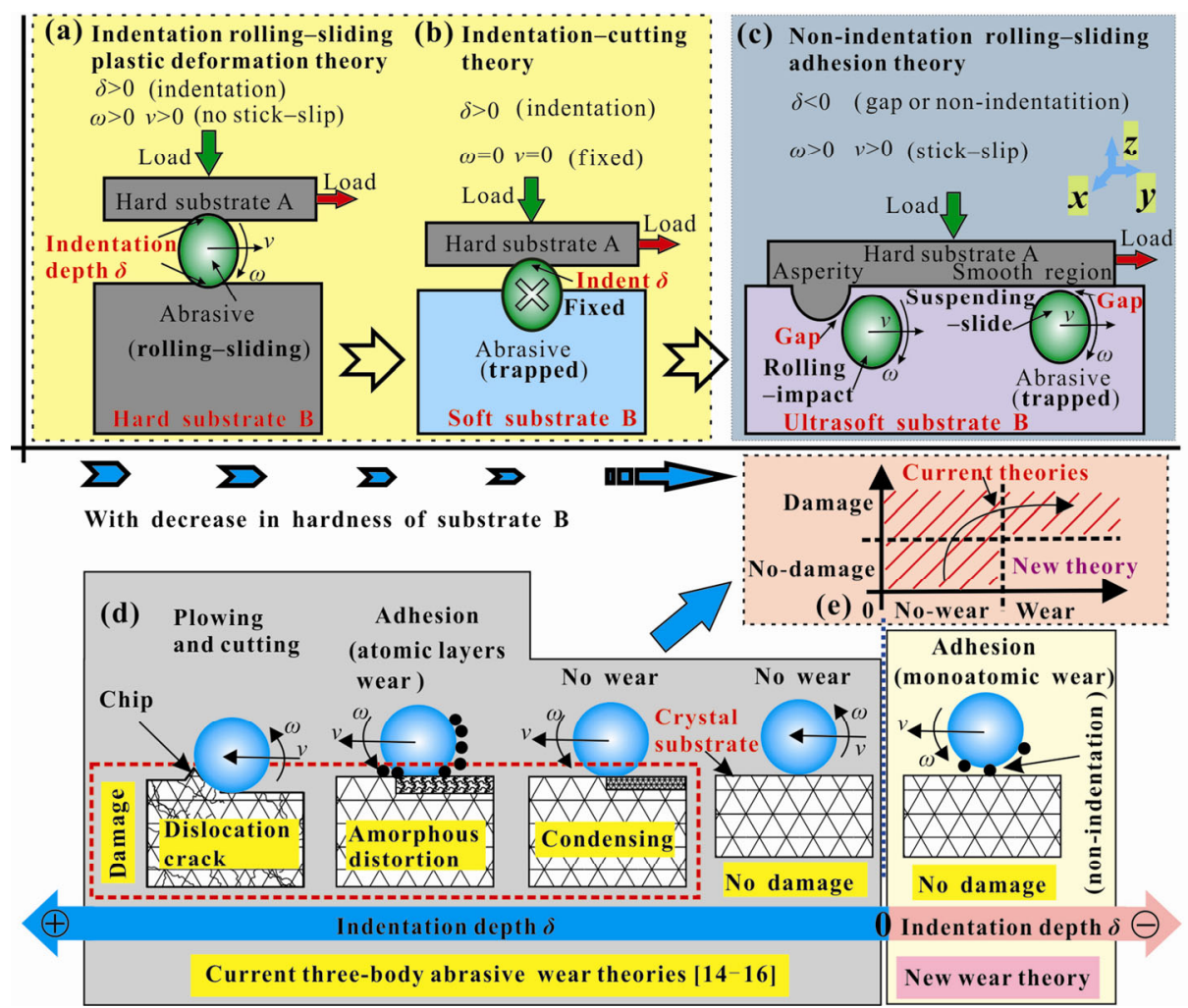

Fig. 6 Schematic diagrams of evolution of three-body abrasive wear mechanism. (a) Existing indentation rolling-sliding plastic deformation model for three-body abrasive wear between hard/hard mating surfaces. (b) Existing indentation-cutting model for three-body abrasive wear between soft/hard mating surfaces. (c) New non-indentation rolling-sliding adhesion model for three-body nano-abrasive wear between ultrasoft/hard mating surfaces. (d) Wear regimes of single crystal substrate under three-body abrasive wear. (e) Wear-damage regime diagram based on different wear theories.

\subsection{New abrasive motion state}

The abrasive motion state of the new theory should be a new form that differs from known abrasive motion states. The abrasives are trapped in the soft substrate and will be stationary in the three-body abrasive wear between the soft/hard mating surfaces (Fig. 6(b)), whereas the abrasives will exhibit continuous rollingsliding without stick-slip in three-body abrasive wear between hard/hard mating surfaces (Fig. 6(a)). The abrasive motion state of rolling-sliding with stick-slip accompanied by trapping in the ultrasoft substrate should be the intermediate status between the two known abrasive motion states, as shown in Fig. 6(c).

\subsection{New wear regime}

The new wear theory claims a new wear regime for three-body abrasive wear. As shown in Fig. 6(d), the current three-body or two-body abrasive wear theories $[11,14-16]$ suggest that as the depth of indentation of the abrasives into the hard mating substrate increases gradually from zero, various types of damage will occur in hard crystal substrates, such as amorphous and condensing damages. Once the damage above accumulates to a certain degree, various types of material wear (e.g., adhesive wear, plow wear, and cutting wear) and new damages (i.e., dislocations and cracks) will appear on the surface of the hard substrate. In other words, as the indentation depth of the abrasive increases gradually from zero, the substrate will undergo three regimes in sequence, i.e., a no-wear and no-damage regime, a no-wear and damage regime, and a wear and damage regime. However, the new wear mechanism of this study shows that wear and no-damage regimes will occur when the abrasive is in a non-indentation state. Therefore, a wear-damage regime diagram based on different wear theories was established, as shown in Fig. 6(e).This new theory can be supported by experimental phenomena of scanning probe lithography [38]. 
The adhesive wear process between the new theory and the existing three-body abrasive wear theory differs. In the adhesive wear process based on existing theories, material removal is in the form of a single atomic layer or multiple atomic layers. Therefore, the worn substrate is typically accompanied by various types of damages, such as amorphization or lattice distortion within several surface atomic layers. However, in the adhesive wear process based on the new wear theory, material is removed monoatomically. Therefore, the substrate surface will not be damaged. This new theory is beneficial for improving the theory of atomic machining or atomic manufacturing [39]. Atomic machining can be defined as a process in which a single atom or several atoms are removed from the surface of a workpiece after each contact event between the tool and workpiece. To achieve atomic machining, the real working load acting on the tool and the real contact radius between the tool and workpiece must be sufficiently low. In CMP, although the size and nominal operating load of the abrasive are at the nanometer or micron scale, the real operating load and real contact radius of the abrasive are below the nanometer scale; hence, the atomic machining is enabled.

It is clear that the indentation-plowing process is not necessary for material removal during CMP. The abrasive must penetrate the surface of the hard substrate to realize material removal from the hard substrate surface, according to existing three-body abrasive theories and the corresponding indentationplowing model of CMP. However, based on the new non-indentation wear mechanism, the monoatomic damage-free material removal process on a hard surface can be achieved by the adhesion between the abrasive and hard surface when the abrasive is suspended on the surface of the hard substrate. This new theory explains the non-existence of scratches on the wafer surface under normal CMP operating conditions. Furthermore, it explains why CMP technology can always satisfy the process requirements of IC manufacturing, even when the line width of the ICs is reduced to $7 \mathrm{~nm}$ or less.

\subsection{New material removal mechanism of CMP}

This new theory claims that the abrasives will slide in a suspended state in the smooth region of the wafer and undergo rolling impact at the asperity region of the wafer, unifying the current mainstream CMP material removal theories. In the smooth region of a wafer or silicon substrate, the suspension sliding of the abrasive is similar to the indentation-plowing process of the abrasive at almost zero indentation depth $[15,16]$. Moreover, as shown in Fig. 5(b), the material removal volume was insensitive to the operating load, consistent with the Zhao model [25] of indentation-plowing theory. However, in the asperity region of a wafer or silicon substrate, the rolling impact process of the abrasive was similar to the horizontal impact process of the abrasive [27]; hence, the problem involving the initial incident energy source of abrasives in the existing abrasive-impact model of CMP material removal can be solved. Moreover, the material removal volume increased with the operating load, consistent with the Luo model [23] of indentation-plowing theory. Hence, during $\mathrm{CMP}$, the interaction between the abrasives and wafer cannot be described by a simple indentation-plowing process or a simple abrasive-impact process. This new theory not only solves the current conflict between indentation-plowing and abrasive-impact theories, but also rectifies the discrepancy between the Zhao and Luo models for indentation-plowing theory.

In addition, the corresponding material removal rate (MRR) can be expressed as

$$
\begin{gathered}
\mathrm{MRR}=\mathrm{MRR}_{1}+\mathrm{MRR}_{2} \\
=f_{1}\left(L F_{z}, E_{\mathrm{p}}, H_{\mathrm{w}}, d_{\mathrm{a}}, \ldots\right) \cdot D V_{y}+f_{2}\left(E_{\mathrm{p}}, H_{\mathrm{w}}, d_{\mathrm{a}}, \ldots\right) \cdot D V_{y}
\end{gathered}
$$

If the entire sliding distance is in a rough or smooth state, then the material removal rate is expressed as $\mathrm{MRR}_{1}$ or $\mathrm{MRR}_{2}$, respectively. In addition to being related to the elastic modulus of the pad $\left(E_{\mathrm{p}}\right)$, wafer hardness $\left(H_{\mathrm{w}}\right)$, and abrasive size $\left(d_{\mathrm{a}}\right), \mathrm{MRR}_{1}$ increases with the load $\left(L F_{z}\right)$ and relative speed $\left(D V_{y}\right)$ (Fig. 5(c)). However, $\mathrm{MRR}_{2}$ is insensitive to the load $\left(L F_{z}\right)$.

\section{Conclusions}

The non-indentation rolling-sliding adhesion theory proposed herein is a new, third type of wear mechanism for three-body abrasive wear. According to this theory, 
non-damaging monoatomic material wear can be realized when the abrasive is in a non-indentation state, thereby completing the theory of atomic machining or atomic manufacturing. This provides further opportunities for investigating three-body abrasive wear and eventually promotes the establishment of monoatomic-scale three-body abrasive wear theory.

Our current study also showed that the indentationplowing process is not necessary for achieving material removal during CMP. In fact, the abrasives should slide in a suspended state in the smooth region of the wafer and undergo rolling impact at the asperity region of the wafer during CMP. The confusion between the existing indentation-plowing theory and abrasiveimpact theory is primarily due to the incorrect basic contact theory model of abrasives. The non-indentation rolling-sliding adhesion material removal mechanism is novel and provides revolutionary understanding regarding the $\mathrm{CMP}$ microscopic material removal process, challenging the current understanding of the CMP material removal mechanism that has existed for the past 30 years. This new mechanism will significantly affect the research and development of new CMP technologies, particularly for CMP consumables.

\section{Acknowledgements}

We acknowledge Dr. Ji'nan DENG in Chongqing University for her help in the process of writing this manuscript. This work was supported by the National Natural Science Foundation of China (Nos. 51375291 and 91323302) and the Natural Science Foundation of Shanghai (No. 19ZR1401500).

Electronic Supplementary Material Supplementary material is available in the online version of this article at https://doi.org/10.1007/s40544-020-0481-1.

Open Access This article is licensed under a Creative Commons Attribution 4.0 International License, which permits use, sharing, adaptation, distribution and reproduction in any medium or format, as long as you give appropriate credit to the original author(s) and the source, provide a link to the Creative Commons licence, and indicate if changes were made.
The images or other third party material in this article are included in the article's Creative Commons licence, unless indicated otherwise in a credit line to the material. If material is not included in the article's Creative Commons licence and your intended use is not permitted by statutory regulation or exceeds the permitted use, you will need to obtain permission directly from the copyright holder.

To view a copy of this licence, visit http://creativecommons.org/licenses/by/4.0/.

\section{References}

[1] Bingley M S, Schnee S. A study of the mechanisms of abrasive wear for ductile metals under wet and dry three-body conditions. Wear 258: 50-61 (2005)

[2] Adebiyi D I, Popoola A P I. Mitigation of abrasive wear damage of Ti-6Al-4V by laser surface alloying. Mater Des 74: 67-75 (2015)

[3] Singh J, Chauhan A. Overview of wear performance of aluminium matrix composites reinforced with ceramic materials under the influence of controllable variables. Ceram Int 42(1): 56-81 (2016)

[4] Rabinowicz E, Dunn L A, Russell P G. A study of abrasive wear under three-body conditions. Wear 4(5): 345-355 (1961)

[5] Erdemir A, Ramirez G, Eryilmaz O L, Narayanan B, Liao Y F, Kamath G, Sankaranarayanan S K R S. Carbon-based tribofilms from lubricating oils. Nature 536(7614): 67-71 (2016)

[6] Sayfidinov K, Cezan S D, Baytekin B, Baytekin H T. Minimizing friction, wear, and energy losses by eliminating contact charging. Sci $A d v$ 4(11): eaau3808 (2018)

[7] Trezona R I, Allsopp D N, Hutchings I M. Transitions between two-body and three-body abrasive wear: Influence of test conditions in the microscale abrasive wear test. Wear 225-229: 205-214 (1999)

[8] Wang Y L, Wang Z S. An analysis of the influence of plastic indentation on three-body abrasive wear of metals. Wear 122(2): 123-133 (1988)

[9] Rodríguez-Castro G A, Reséndiz-Calderon C D, JiménezTinoco L F, Meneses-Amador A, Gallardo-Hernández E A, Campos-Silva I E. Micro-abrasive wear resistance of $\mathrm{CoB} / \mathrm{Co}_{2} \mathrm{~B}$ coatings formed in CoCrMo alloy. Surf Coat Technol 284: 258-263 (2015)

[10] Yamamoto T, Olsson M, Hogmark S. Three-body abrasive wear of ceramic materials. Wear 174(1-2): 21-31 (1994)

[11] Sun J P, Fang L, Han J, Han Y, Chen H W, Sun K. Abrasive wear of nanoscale single crystal silicon. Wear 307(1-2): 119-126 (2013) 
[12] Mishra V, Biswas S. Three-body abrasive wear behavior of short jute fiber reinforced epoxy composites. Polym Compos 37(1): 270-278 (2016)

[13] Yousif B F, Nirmal U, Wong K J. Three-body abrasion on wear and frictional performance of treated betelnut fibre reinforced epoxy (T-BFRE) composite. Mater Des 31(9): 4514-4521 (2010)

[14] Ewen J P, Gattinoni C, Thakkar F M, Morgan N, Spikes H A, Dini D. Nonequilibrium molecular dynamics investigation of the reduction in friction and wear by carbon nanoparticles between iron surfaces. Tribol Lett 63(3): 38 (2016)

[15] Zhang L C, Tanaka H. Atomic scale deformation in silicon monocrystals induced by two-body and three-body contact sliding. Tribol Int 31(8): 425-433 (1998)

[16] Komanduri R, Chandrasekaran N, Raff L M. Molecular dynamics simulation of atomic-scale friction. Phys Rev B 61(20): 14007 (2000)

[17] Feng X D, Sayle D C, Wang Z L, Paras M S, Santora B, Sutorik A C, Sayle T X T,Yang Y, Ding Y, Wang X D, et al. Converting ceria polyhedral nanoparticles into single-crystal nanospheres. Science 312(5779): 1504-1508 (2006)

[18] Chen H, Guo D, Xie G X, Pan G S. Mechanical model of nanoparticles for material removal in chemical mechanical polishing process. Friction 4(2): 153-164 (2016)

[19] Kim B S, Tucker M H, Kelchner J D, Beaudoin S P. Study on the mechanical properties of CMP pads. IEEE Trans Semicond Manufact 21(3): 454-463 (2008)

[20] Hopcroft M A, Nix W D, Kenny T W. What is the Young's modulus of silicon? J Microelectromech Syst 19(2): 229-238 (2010)

[21] Qin K D, Moudgil B, Park C W. A chemical mechanical polishing model incorporating both the chemical and mechanical effects. Thin Solid Films 446(2): 277-286 (2004)

[22] Ye Y, Biswas R, Bastawros A, Chandra A. Simulation of chemical mechanical planarization of copper with molecular dynamics. Appl Phys Lett 81(10): 1875-1877 (2002)

[23] Luo J F, Dornfeld D A. Material removal mechanism in chemical mechanical polishing: Theory and modeling. IEEE Trans Semicond Manufact 14(2): 112-133 (2001)

[24] Fu G H, Chandra A, Guha S, Subhash G. A plasticity-based model of material removal in chemical-mechanical polishing (CMP). IEEE Trans Semicond Manufact 14(4): 406-417 (2001)

[25] Zhao Y W, Chang L. A micro-contact and wear model for chemical-mechanical polishing of silicon wafers. Wear 252(3-4): 220-226 (2002)

[26] Chen R L, Li S X, Wang Z, Lu X C. Mechanical model of single abrasive during chemical mechanical polishing:
Molecular dynamics simulation. Tribol Int 133: 40-46 (2019)

[27] Chagarov E, Adams J B. Molecular dynamics simulations of mechanical deformation of amorphous silicon dioxide during chemical-mechanical polishing. J Appl Phys 94(6): 3853-3861 (2003)

[28] Han X S, Gan Y X. Analysis the complex interaction among flexible nanoparticles and materials surface in the mechanical polishing process. Appl Surf Sci 257(8): 3363-3373 (2011)

[29] Chen R L, Jiang R R, Lei H, Liang M. Material removal mechanism during porous silica cluster impact on crystal silicon substrate studied by molecular dynamics simulation. Appl Surf Sci 264: 148-156 (2013)

[30] Guo Q, Izumisawa S, Phillips D M, Jhon M S. Surface morphology and molecular conformation for ultrathin lubricant films with functional end groups. J Appl Phys 93(10): 8707-8709 (2003)

[31] Castillo-Mejia D, Gold S, Burrows V, Beaudoin S. The effect of interactions between water and polishing pads on chemical mechanical polishing removal rates. $J$ Electrochem Soc 150(2): G76 (2003)

[32] Watanabe T, Fujiwara H, Noguchi H, Hoshino T, Ohdomari I. Novel interatomic potential energy function for $\mathrm{Si}, \mathrm{O}$ mixed systems. Jpn J Appl Phys 38(4A): L366-L369 (1999)

[33] Evans D J, Hoover W G, Failor B H, Moran B, Ladd A J C. Nonequilibrium molecular dynamics via Gauss's principle of least constraint. Phys Rev A 28(2): 1016 (1983)

[34] Budnitzki M, Kuna M. A thermomechanical constitutive model for phase transformations in silicon under pressure and contact loading conditions. Int J Solids Struct 49(11-12): 1316-1324 (2012)

[35] Si L N, Guo D, Luo J B, Lu X C, Xie G X. Abrasive rolling effects on material removal and surface finish in chemical mechanical polishing analyzed by molecular dynamics simulation. J Appl Phys 109(8): 084335 (2011)

[36] Gotsmann B, Lantz M A. Atomistic wear in a single asperity sliding contact. Phys Rev Lett 101(12): 125501 (2008)

[37] Bun-Athuek N, Yoshimoto Y, Sakai K, Khajornrungruang $\mathrm{P}$, Suzuki K. Study on effect of the surface variation of colloidal silica abrasive during chemical mechanical polishing of sapphire. Jpn J Appl Phys 56: 07KB01 (2017)

[38] Chen L, Wen J L, Zhang P, Yu B J, Chen C, Ma T B, Lu X C, Kim S H, Qian L M. Nanomanufacturing of silicon surface with a single atomic layer precision via mechanochemical reactions. Nat Commun 9: 1542 (2018)

[39] Fang F Z, Zhang N, Guo D M, Ehmann K, Cheung B, Liu $\mathrm{K}$, Yamamura K. Towards atomic and close-to-atomic scale manufacturing. Int J Extrem Manuf 1(1): 012001 (2019) 


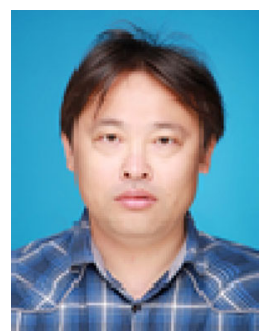

Ruling CHEN. He received his Ph.D. degree from Tsinghua University, China, in 2009, majoring in mechanical engineering. He is now an associate professor at Donghua University, China. His research areas include nanotribology, chemical mechanical polishing (CMP), and ultra-precision surface machining. 